neurocircuits: implications for the pathophysiology of Parkinson's disease, schizophrenia and attention deficit hyperactivity disorder. Neurotox. Res. 10:167-179.

4. Yao, W.-D., Spealman, R.D., and Zhang, J. 2008 Dopaminergic signaling in dendritic spines. Biochem. Pharmacol. 75:2055-2069.

5. He, L., Vasiliou, K., and Nebert, D.W. 2009. Analysis and update of the human solute carrier (SLC) gene superfamily. Hum. Genomics. 3:195-206.

6. Torres, G.E., and Amara, S.G. 2007. Glutamate and monoamine transporters: new visions of form and function. Curr. Opin. Neurobiol. 17:304-312.

7. Yamashita, A., Singh, S.K., Kawate, T., Jin, Y., and Gouaux, E. 2005. Crystal structure of a bacterial homologue of $\mathrm{Na}^{+} / \mathrm{Cl}^{-}$-dependent neurotransmitter transporters. Nature. 437:215-223.

8. Krause, J. 2008. SPECT and PET of the dopamine transporter in attention-deficit/hyperactivity disorder. Expert Rev. Neurother. 8:611-625.

9. Gainetdinov, R.R. 2008. Dopamine transporter mutant mice in experimental neuropharmacology. Naunyn Schmiedebergs Arch. Pharmacol. 377:301-313.

10. Li, L.-B., et al. 2004. The role of N-glycosylation in function and surface trafficking of the human dopamine transporter. J. Biol. Chem. 279:21012-21020.

11. Miranda, M., Dionne, K.R., Sorkina, T., and Sorkin, A. 2007. Three ubiquitin conjugation sites in the amino terminus of the dopamine transporter mediate protein kinase C-dependent endocytosis of the transporter. Mol. Biol. Cell. 18:313-323.

12. Norregaard, L., Loland, C.J., and Gether, U. 2003. Evidence for distinct sodium-, dopamine-, and cocaine-dependent conformational changes in transmembrane segments 7 and 8 of the dopamine transporter. J. Biol. Chem. 278:30587-30596.

13. Mazei-Robison, M.S., et al. 2008. Anomalous dopamine release associated with a human dopamine transporter coding variant. J. Neurosci. 28:7040-7046.

14. Kurian, M.A., et al. 2009. Homozygous loss-of-function mutations in the gene encoding the dopamine transporter are associated with infantile parkinson- ism-dystonia. J. Clin. Invest. 119:1595-1603.

15. Zafeiriou, D.I., et al. 2009. Tyrosine hydroxylase deficiency with severe clinical course. Mol. Genet. Metab. 97:18-20.

16. Assmann, B.E., et al. 2004. Infantile parkinsonismdystonia and elevated dopamine metabolites in CSF. Neurology. 62:1872-1874.

17. Lander, E.S., and Botstein, D. 1987. Homozygosity mapping: a way to map human recessive traits with the DNA of inbred children. Science. 236:1567-1570.

18. Gibbs, J.R., and Singleton, A. 2006. Application of genome-wide single nucleotide polymorphism typing: simple association and beyond. PLoS Genet. 2:e150

19. Nikolaus, S., et al. 2007. Investigating the dopaminergic synapse in vivo. I. Molecular imaging studies in humans. Rev. Neurosci. 18:439-472.

20. Nikolaus, S., et al. 2007. Investigating the dopaminergic synapse in vivo. II. Molecular imaging studies in small laboratory animals. Rev. Neurosci. 18:473-504.

\title{
Can childhood viral infection protect from type 1 diabetes?
}

\author{
Terry B. Strom
}

Beth Israel Deaconess Medical Center, Boston, Massachusetts, USA.

\begin{abstract}
While many candidate type 1 diabetes (T1D) susceptibility genes have been identified, evidence suggests that environmental stimuli, such as viral infections, may also be involved in T1D pathogenesis. However, how viral infections may prevent or trigger the diabetogenic process remains unclear. In this issue of the JCI, Filippi et al. show that infection of NOD mice with Coxsackie virus B3 or lymphocytic choriomeningitis virus, neither of which directly destroys insulin-secreting pancreatic $\beta$ cells, triggers the activation of two distinct immunoregulatory mechanisms, involving both the innate and adaptive immune system, that protect against the development of T1D in these animals (see the related article beginning on page 1515).
\end{abstract}

Immune-mediated destruction of insulin-secreting pancreatic $\beta$ cells results in type 1 diabetes (T1D). Genetic factors, including genes that regulate facets of the immune response, influence the susceptibility of an individual to T1D and other autoimmune diseases. Yet the concordance rate for T1D among monozygotic twins is only $40 \%$ (reviewed in ref. 1). Therefore, environmental or epigenetic factors must exist that trigger or mediate resistance to the development of overt T1D in geneti-

Conflict of interest: The author is a consultant for Quest Diagnostics Inc.

Nonstandard abbreviations used: CVB3, Coxsackie virus B3; IGRP, islet-specific glucose-6-phosphate catalytic-related protein; LCMV, lymphocytic choriomeningitis virus; PD-1, programmed cell death-1; PD-L1, PD-1 ligand 1; T1D, type 1 diabetes.

Citation for this article: J. Clin. Invest. 119:1458-1461 (2009). doi:10.1172/JCI39565. cally predisposed individuals. Among the environmental factors that might influence T1D expression, childhood infectious diseases have received considerable attention. Why? Clonotypic $\mathrm{T}$ cell receptors that recognize certain microbial antigens cross-react with self antigens. Some viruses can infect and damage pancreatic $\beta$ cells. Hence, $\mathrm{T}$ cell clones activated by microbes can, on occasion, cross-react with molecularly related self antigens (a process known as molecular mimicry) and precipitate overt autoimmune disease. However, epidemiologic data do not support a link between childhood infection and autoimmune disease (reviewed in ref. 1). Indeed, developed countries, which have a low incidence of childhood infection, have a high rate of autoimmune disease, while the reverse is true for less-developed counties, which have a high rate of childhood infection (1).
As persons from a less-developed region of a country with a low rate of autoimmune disease and high rates of childhood infection migrate to a more developed region of the country with lower rates of childhood infection, the incidence of autoimmune diseases among the migrants and their offspring reciprocally increases (1). Furthermore, it has been previously reported in rodent models of T1D, such as the NOD mouse, that exposure to infectious agents can provide powerful protection from T1D in these animals (reviewed in 1). However, how infections protect against autoimmune disease is unknown. In their current study in this issue of the JCI, Filippi et al. (2) demonstrate that infection of prediabetic NOD mice with Coxsackie virus B3 (CVB3) or lymphocytic choriomeningitis virus (LCMV) - pancreatotropic viruses that are known to cause systemic infection but do not lyse pancreatic $\beta$ cells - reduces the incidence and delays the onset of T1D in these animals. The authors demonstrate that viral infection provides protection from T1D by triggering distinct immunoregulatory mechanisms, involving both the innate and adaptive immune systems, that prevent the expansion of diabetogenic $T$ cells.

The inception and termination of $\mathrm{T}$ celldependant antiviral immune and autoimmune responses are orchestrated through 


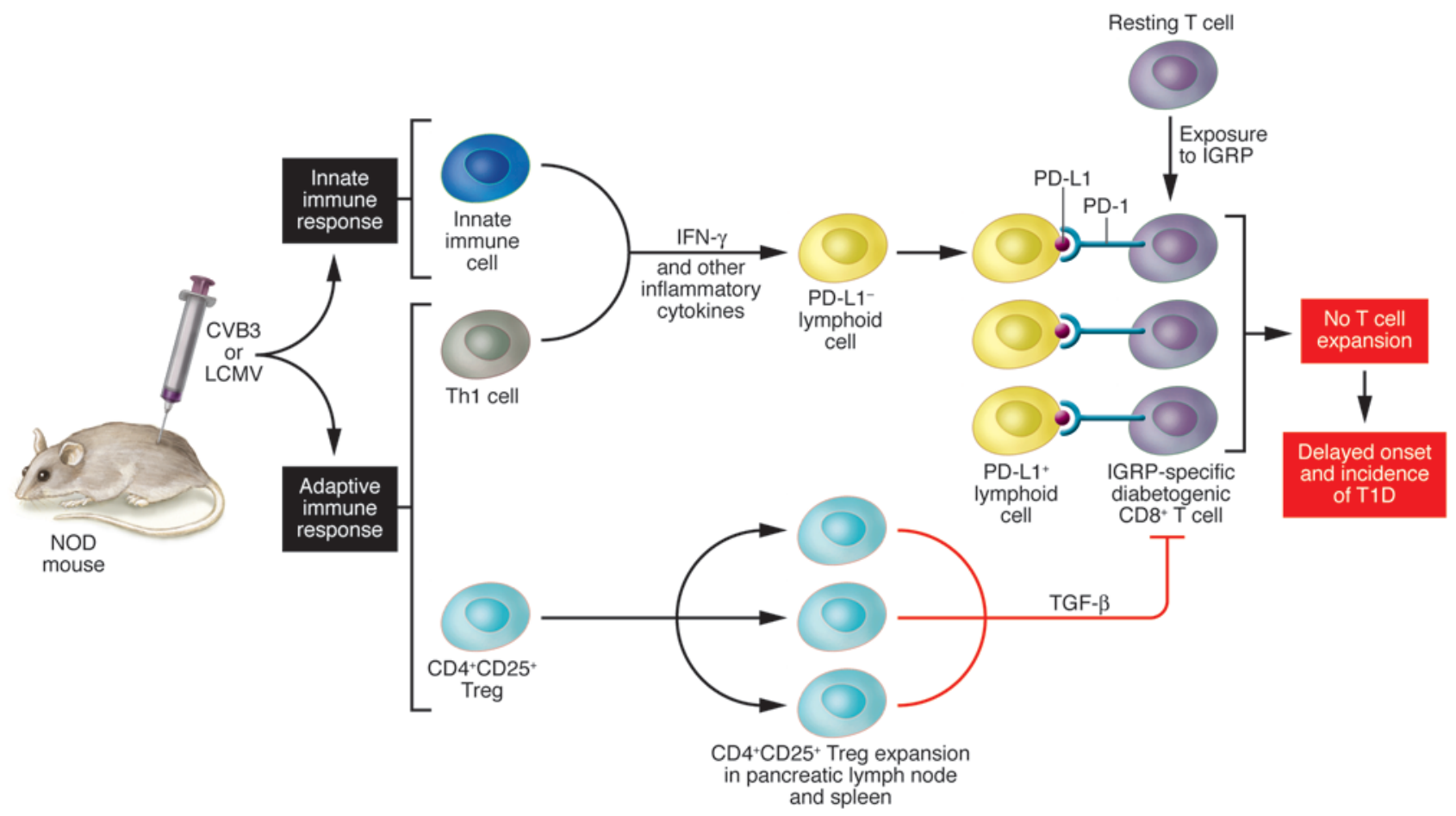

Figure 1

Immunoregulatory mechanisms induced by viral infection protect against T1D in mice. In their study in this issue of the $\mathrm{JCl}$, Filippi et al. examined the mechanisms by which infection with CVB3 or LCMV (which do not directly destroy insulin-producing pancreatic $\beta$ cells) reduced the incidence and delayed the onset of T1D in NOD mice (2). The authors report that immune cells from both the innate and adaptive immune system are involved in curbing the expansion of autoaggressive T cells that target $\beta$ cells. They show that IFN- $\gamma$ produced by adaptive Th1 cells, as well as by various innate immune cells in response to viral infection, upregulates the expression of PD-L1 on a varied population of lymphoid cells. Following interaction with $\beta$ cell antigen IGRP, activated autoaggressive T cells express the PD-L1 receptor PD-1. PD-1+ T cells and PD-L1+ cells occupy overlapping habitats. The cognate interaction of PD-1 with PD-L1 inhibits the expansion of IGRP-specific, diabetogenic PD-1+ CD8+ T cells, which delays the onset of T1D. CD4 ${ }^{+} \mathrm{CD} 25^{+}$Tregs, which are part of the adaptive immune system, are activated by both viruses and autoantigens. These cells produce TGF- $\beta$, a potent immunosuppressive cytokine. Filippi et al. also show that after CVB3 or LCMV infection in NOD mice, the populations of Tregs in the pancreatic lymph node and spleen were increased. Production of TGF- $\beta$ by these cells curbed the expansion of autoaggressive T cells and limited their cytodestructive properties, thereby protecting insulin-producing $\beta$ cells and reducing the incidence of T1D in these animals. The physical proximity of regulatory and cytodestructive autoimmune cells enables immunoregulation.

similar pathways in which inflammatory innate and antigen-specific adaptive immunity interact. The balance of proand antiinflammatory cytokines present within the milieu in which self or microbial antigens stimulate the activation of naive $\mathrm{CD}^{+} \mathrm{T}$ cells dictates whether newly activated $\mathrm{CD}^{+} \mathrm{T}$ cells commit to a tissue-destructive (e.g., Th1, Th17) or tissueprotective immunoregulatory (i.e., Treg) phenotype (3). Inflammatory cytokines also influence the phenotype of antigenactivated $\mathrm{CD}^{+} \mathrm{T}$ cells; however, this topic has received less attention. Among CD $4^{+}$ $\mathrm{T}$ cells, proinflammatory Th17 cells are particularly adept at effecting tissue injury (4), as exemplified in autoimmune conditions including T1D (5). TGF- $\beta$, a cytokine that has immunosuppressive properties and induces development of the immunoregulatory Treg phenotype in newly acti- vated $\mathrm{CD}^{+} \mathrm{T}$ cells (6), is shown in the current study by Filippi et al. (2) to be robustly expressed by Tregs harvested from NOD mice bearing protective pancreatotropic viruses (Figure 1). Yet it is well known that hyperexpression of TGF- $\beta$ does not invariably lead to heightened immunoregulatory activity. TGF- $\beta$ coexpressed in an environment with an abundance of proinflammatory cytokines such as IL- 6 or IL-21 directs newly activated $\mathrm{CD}^{+} \mathrm{T}$ cells to develop the highly cytopathic Th17 phenotype (7-9). Indeed, the presence of IL- 6 or IL-21 totally negates the potential to instruct naive $\mathrm{CD}^{+} \mathrm{T}$ cells to develop a tissue-protective Treg phenotype (7-9). In short, the commitment of $\mathrm{CD}^{+} \mathrm{T}$ cells to a highly tissue-destructive or to a tissue-protective $\mathrm{T}$ cell phenotype hinges on the fine balance between select pro- and antiinflammatory cytokines present within the context of antigen activation. The exquisite interplay between adaptive and innate immunity is also responsible for terminating $\mathrm{T}$ celldependent reactions in vivo.

\section{Virus-triggered IFN-Y secretion limits expansion of $\beta$ cell- destructive $\mathrm{T}$ cells}

Filippi et al. (2) also observed that, after interaction with a major islet cell autoantigen (islet-specific glucose-6-phosphate catalytic-related protein [IGRP]), IGRP-specific cytotoxic $\mathrm{CD}^{+} \mathrm{T}$ cells became activated and expressed programmed cell death-1 (PD-1) proteins on their cell surface, which renders these cells sensitive to an immunoinhibitory signal that results from ligation of PD- 1 by PD-1 ligand 1 (PD-L1) (10) (Figure 1). Thus, $\mathrm{T}$ cell activation, a process that is necessary for expression of effector $\mathrm{T}$ cell tissue-destructive properties, also 
rendered these cells sensitive to $\mathrm{PD}-1$-mediated immunoinhibitory signals. Filippi et al. also show that infection of prediabetic NOD mice with CVB3 or LCMV resulted in the hyperexpression of the proinflammatory cytokine IFN- $\gamma$ by adaptive Th 1 cells as well as by various innate immune cells, including microvascular endothelial cells and mononuclear leukocytes (Figure 1). The authors observed that interaction of PD-L $1^{+}$cells with PD- $1^{+}$IGRP-specific cytotoxic $\mathrm{CD}^{+} \mathrm{T}$ cells sharply limited expansion of these $\beta$ cell-destructive PD-L1 ${ }^{+}$ $\mathrm{T}$ cells, which delayed the onset of T1D in the NOD mice infected with non-lytic CVB3 or LCMV pancreatotropic viruses (2). Hence, a negative feedback loop that rests upon the coordinated consequences of $\mathrm{T}$ cell activation and presence of an inflammatory cytokine springs into action.

\section{TGF- $\beta$ restrains the $\beta$ cell- destructive properties of autoreactive T cells}

Filippi et al. also show that a second mechanism of immunoregulation amplified in CVB3- or LCMV-infected NOD mice protects the mice from T1D (2). The authors report that while Tregs activated by viral infection do not recognize islet-specific autoantigens, infection with either of these two T1D-protective viruses magnified the ability of Tregs to prevent T1D (2). Why? Tregs are able to curtail the expansion and activity of tissue-destructive $T$ cells through a variety of mechanisms $(6,11)$. In some cases, immunoregulation is exerted via non-antigen-specific means, for example, the production of immunosuppressive cytokines such as IL-10 or TGF- $\beta$ (11), or by adenosine, another type of immunosuppressive molecule (12). The enhanced capacity of Tregs from NOD animals infected with the T1D-protective viruses CVB3 or LCMV, as compared with Tregs from uninfected control NOD mice, to block diabetes is shown by Filippi et al. to be mediated by amplified TGF- $\beta$ expression by Tregs (2) (Figure 1). The Treg populations in the pancreatic lymph node and spleen were also shown to be increased in the CVB3- or LCMV-infected animals. Thus, amplified TGF- $\beta$ production by CVB3- or LCMV-reactive Tregs and/or APCs residing in the same microenvironment through which effector $\mathrm{T}$ cells reactive to $\beta$ cell autoantigens traverse exerts effective, albeit non-antigenspecific, immunoregulatory effects within this environment. In this context, CVB3or LCMV-reactive Tregs, which develop as part of the adaptive immune response, act in a non-antigen-specific manner to block the autoimmunity mediated by autoantigen-responsive T cells. In summary, the strengthened immunoregulatory networks evident in NOD mice infected with T1Dprotective viruses synergize to block T1D development by restraining the expansion of autoreactive T cells via (a) the interaction of PD- $1^{+}$autoimmune cells with an IFN- $\gamma$-dependent expanded population of PD-L $1^{+}$cells and (b) the TGF- $\beta$-dependent amplification of Treg-dependent immunoregulation (Figure 1).

\section{Regional, virus-induced immunoregulation prevents T1D}

Nature has provided means to curtail prolonged tissue-destructive immune and inflammatory processes. The consequences of overly prolonged cytodestructive immunoinflammatory reactions are so dire that effective and multitiered feedback regulation is imperative. In their current study, Filippi et al. reveal that antiviral immunity and autoimmunity, coordinated in time and location, can interact and conspire to block the clinical expression of T1D in NOD mice (2). In essence, antiviral and autoimmune $\mathrm{T}$ cells can occupy overlapping habitats. The $\mathrm{T}$ cell-dependent antiviral immune response, characterized by marked inflammation in the absence of $\beta$ cell injury, evokes naturally occurring feedback regulation of virus-reactive effector $T$ cells. Insofar as this immunoregulatory response to terminate tissue-destructive antiviral immunity is mounted within a habitat also bearing cellular constituents of the autoimmune response, heightened naturally occurring feedback inhibition - created to control both colocalized antiviral immunity and (probably) the autoimmune response serves to efficiently abort tissue-destructive forms of $\beta$ cell-directed autoimmunity.

Tregs are the primary cell type responsible for creating immune tolerance, a state in which tissue antigen-specific protective $\mathrm{T}$ cells restrain the antigen-specific tissue effector $\mathrm{T}$ cells from destroying tissues that bear antigens that are recognized by these different $T$ cell subsets. In most situations in which immune tolerance has been analyzed, Tregs and tissue-destructive T cells recognize antigens expressed on the same tissue. As Filippi et al. show (2), in NOD mice infected with T1D-preventing viruses, $\beta$ cells are protected from immune-mediated injury through the additive effects of $\beta$ cell-specific and non- $\beta$ cell-reactive, virus-specific $T$ cells. The findings of this new study emphasize the potential for developing new vaccination strategies in which cells adjacent to cells at risk for autoimmune injury are targeted for infection by select noncytopathic viruses that foster immunoprotection mediated by Tregs that home to the same microenvironment as cells targeted for immune injury. Regional, not necessarily antigen-specific, immunoprotection may be sufficient to prevent T1D and other autoimmune diseases, even in the numerous circumstances in which the antigen(s) that trigger the autoimmune state in the afflicted individual are not known. Unfortunately, there are complexities that must be surmounted. Some pancreatotropic viruses, for example, CVB4, exert diabetogenic, not tolerogenic, effects in NOD mice (13). For such a vaccination strategy to prove effective, it will be necessary to develop vaccines that target the appropriate tissues and also elicit expression of immunoinhibitory cytokines such as TGF- $\beta$ in the absence of cytokines that direct $T$ cells to develop the tissuedestructive Th17 phenotype.

\section{Acknowledgments}

The author is supported by grants from the Juvenile Diabetes Research Foundation (4-2007-1054) and the NIH (5-P01 AI 041521-12).

Address correspondence to: Terry B. Strom, Beth Israel Deaconess Medical Center, 330 Brookline Avenue, E/CLS 608, Boston, Massachusetts 02215, USA. Phone: (617) 735-2880 Fax: (617) 667-0923; E-mail: tstrom@bidmc.harvard.edu.

1. Bach, J.-F. 2002. The effect of infections on susceptibility to autoimmune and allergic diseases. N. Engl. J. Med. 347:911-920.

2. Filippi, C.M., Estes, E.A., Oldham, J.E., and von Herrath, M.G. 2009. Immunoregulatory mechanisms triggered by viral infections protect from type 1 diabetes in mice. J. Clin. Invest. 119:1515-1523.

3. Dong, C. 2008. TH17 cells in development: an updated view of their molecular identity and genetic programming. Nat. Rev. Immunol. 8:337-348.

4. O'Quinn, D.B., Palmer, M.T., Lee, Y.K., and Weaver, C.T. 2008. Emergence of the Th17 pathway and its role in host defense. Adv. Immunol. 99:115-163.

5. Jain, R., et al. 2008. Innocuous IFNgamma induced by adjuvant free agents restores normoglycemia in NOD mice through inhibition of IL-17 production. J. Exp. Med. 205:207-218.

6. Sakaguchi, S., Yamaguchi, T., Nomura, T., and Oto, M. 2008. Regulatory T cells and immune tolerance. Cell. 133:775-787.

7. Bettelli, E., et al. 2006. Reciprocal developmental pathways for the generation of pathogenic effector TH17 and regulatory T cells. Nature. 441:235-238.

8. Veldhoen, M., et al. 2006. TGFbeta in the context of an inflammatory cytokine milieu supports de 
novo differentiation of IL-17 producing T cells. Immunity. 24:179-189.

9. Kom, T., et al. 2007. IL-21 initiates an alternative pathway to induce proinflammatory TH17 cells. Nature. 448:484-487.

10. Keir, M.E., Butte, M.J., Freeman, G.J., and Sharpe,
A.H. 2008. PD-1 and its ligands in tolerance and immunity. Annu. Rev. Immunol. 26:677-704.

11. Brusko, T.M., Putman, A.L., and Bluestone, J.A. 2008. Human regulatory T cells: role in autoimmune disease and therapeutic opportunities. Immunol. Rev. 223:371-390.
12. Dwyer, S., et al. 2007. Adenosine catalyzed by CD39 and CD73 expressed on regulatory cells mediates immune suppression. J. Exp. Med. 204:1257-1265.

13. Horwitz, M.S., et al. 1998. Diabetes induced by Coxsackie virus: initiation by bystander damage and not molecular mimicry. Nat. Med. 4:781-785. 